\title{
Pro-angiogenic Effects of a Standardised Herbal Formulation, Sailuotong, on EA. hy926 Cells in vitro and Zebrafish in vivo
}

\author{
Sai Wang Seto ${ }^{1}$, Iris Wai Man Ko${ }^{1}$, Simon Ming Yuen Lee ${ }^{2}$, Hosen Kiat $^{3,4,5}$, Alan Bensoussan ${ }^{1}$, \\ Jianxun Liu ${ }^{6}$, Dennis Chang ${ }^{1}$
}

${ }^{I}$ NICM, Western Sydney University, Australia, ${ }^{2}$ State Key Laboratory Research in Chinese Medicine and Institute of Chinese Medical Sciences, University of Macau, Macao, ${ }^{3}$ Faculty of Medicine, University of New South Wales, Australia, ${ }^{4}$ School of Medicine, Western Sydney University, Australia, ${ }^{5}$ Faculty of Medicine and Health Sciences, Macquarie University, Australia, ${ }^{6} X i y u a n$ Hospital, China Academy of Chinese Medical Sciences, China

Background: Sailuotong (SLT) is a standardised three-herb formula combining specific dosages of Panax ginseng (ginseng), Ginkgo biloba (ginkgo), and Crocus sativus (saffron) for the management of vascular dementia. Recent clinical studies demonstrated that SLT can improve cognitive function and memory in people with vascular dementia. Animal studies have suggested these beneficial effects may be associated with increased cerebral blood circulation. Our study aims to examine the potential pro-angiogenic effect of SLT.

Methods: Cell proliferation (MTT) and cell migration assays were used to evaluate the pro-angiogenic effects of SLT in EA.hy926 cells in vitro. Pharmacological inhibitors, including wortmannin (a phosphatidylinositol 3-kinase (PI3K) inhibitor) and L-N $\omega$-nitro- L-arginine methyl ester hydrochloride (L-NAME) (an endothelial nitric oxide synthase inhibitor) were used to probe the possible involvement of signalling pathways in the SLT-induced EA.hy926 proliferation. Vascular endothelial growth factor (VEGF) tyrosine kinase inhibitor II (VRI)-induced vascular insufficient transgenic Tg (flk1:GFP) zebrafish model was used to test the in vivo pro-angiogenic effects of SLT.

Results: Our results showed that SLT $(6.25-50 \mu \mathrm{g} / \mathrm{ml})$ increased EA.hy926 cell proliferation in a dose-dependent manner. The SLT-induced cell proliferation was sensitive to pre-incubation of wortmannin (100 nM), but not L-NAME $(20 \mu \mathrm{M})$. Similarly, SLT significantly increased the cell migration in the EA.hy926 cell wound healing model $(\mathrm{n}=3)$. SLT $(10-50 \mu \mathrm{g} / \mathrm{ml})$ rescued blood vessel loss in the VRI-induced vascular insufficient Tg(flk1:GFP) zebrafish in a dosedependent manner.

Conclusion:: Our study demonstrated that SLT exhibits a pro-angiogenic effect in both EA.hy926 cells in vitro and VRIinduced vascular insufficient $\mathrm{Tg}(\mathrm{flk} 1 \mathrm{GFP})$ zebrafish in vivo. This effect is possibly mediated via the PI3K signalling pathway. Our findings suggest that the neurological protection and cognitive enhancement effects of SLT observed in animal and clinical studies could at least partially be mediated by its pro-angiogenic property. 